proliferation of the uterine mucosa are enormously stimulated by even small quantities of staltion's urine. After five daily injections each of 0.5 c.c. of stallion's urine, the weight of the uterus of a young rabbit weighing $1200 \mathrm{gm}$. rose from $0.47 \mathrm{gm}$. to $1.8 \mathrm{gm}$. On introducing larger quantities of urine (twelve daily injections each of 3 c.c. of stallion's urine) the weight of the uterus rose from $0.47 \mathrm{gm}$. to $5.48 \mathrm{gm}$. and the weight of the vagina from $0.15 \mathrm{gm}$. to $2 \cdot 48 \mathrm{gm}$. The uterine musculature showed a great increase in muscle cells (hyperplasia), but especially great was the proliferation or what might be more correctly termed the hyperproliferation of the uterine and vaginal mucous membranes. After the injection of stallion's urine there was a retardation of testicular growth in the infantile male rat (antimasculine reaction).

In such chemical properties as have hitherto been observed, the ostrogenic hormone in stallion's urine corresponds with follicular hormone, in which connexion attention is invited to its solubility described above. We are now occupied with the exact characterisation of the hormone.

The details of the investigations and the importance of the reported findings in relation to the biology of the sex hormones will be discussed in a comprehensive publication.

I take this opportunity of expressing my best thanks to Sir Peter Chalmers Mitchell for the gift of material and to Dr. Bernard Homa, of London, for the translation.

Biochemical Institute, University of Stockholm. Jan. 10.

1 Klin. Wochenschr., No. 49, 2285; 1930.

"Die Hormone des Ovariums und des Hypophysenvorderlappens" (The Hormones of the Ovary and of the Anterior Pituitary Lobe). Springer, 1930 , pp. 85 and 90 .

\section{A Source of Error in Photometry}

IN the course of spectroscopic work in this Institute, a source of error in photographic microphotometry has been discussed, which under certain conditions, especially in the case of band spectra, may be rather serious. It is well known that the use of a wide photometer slit causes an error in the determination of the maximum blackening of a spectral line on the plate, the line appearing broader and less intense in the centre than by correct measurement with a narrow slit. It is, however, easily overlooked that an error arises also in the integral intensity of the line, and since the matter, so far as we know, has not been dealt with in the photometric literature, it may be of interest to publish some calculations of the error under various conditions.

For the sake of simplicity, we will assume that the blackening on the plate and the intensity of light are proportional, so that their integrals are equivalent. For the distribution of intensity in the spectral line we choose the Gaussian function $i=i_{0} e^{-k^{2}\left(\nu-v_{0}\right)^{2}}$ ( $i=$ intensity, $\quad v=$ frequency, $k=$ constant). The blackening $S$ is as usual defined by $S=\log J_{0} / J$.

The curve $S$ in Fig. 1 represents the actual blackening on the plate, the curve $J$ in Fig. 2 is the photometer curve of the line when using an infinitely narrow slit. Using a slit of the width $d$ means taking the mean $J^{\prime}$ of the function $J$ in the interval $\nu \pm d / 2$. It is easily seen that the integrals of the curves $J$ and $J^{\prime}$ in Fig. 2 are equal, that is, the area $a$ is equal to the sum of the areas $b$. But when we calculate the blackening $S^{\prime}$ from the formula $S^{\prime}=\log J^{\prime} / J_{0}$, the area $a$ will have a greater weight than the areas $b$, so that the area $A$ in Fig. 1 becomes larger than the sum of the areas $B$. The integral of $S^{\prime}$ will always be smaller than that of $S$.

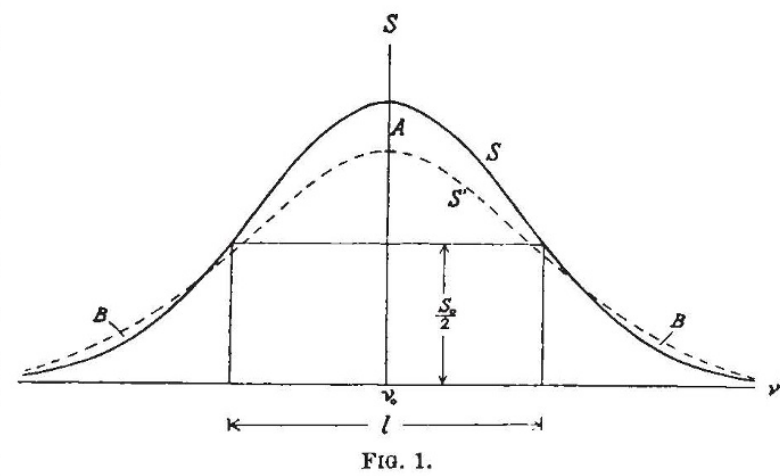

There are two factors which, under the chosen conditions, influence the magnitude of the error, namely, the maximum blackening $S_{0}$ and the ratio

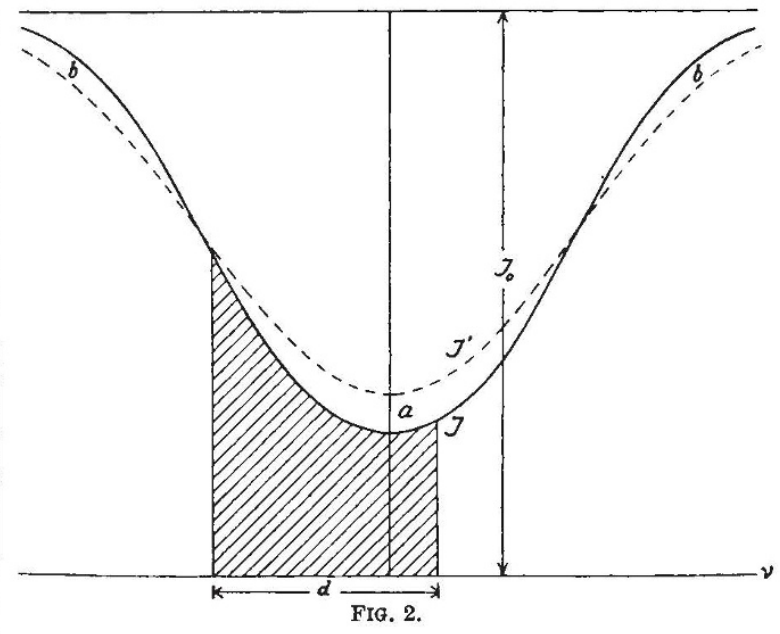

$p=d / l$ ( $l$ is defined in Fig. 1). In the table given below, the error $\int_{-\infty}^{+\infty}\left(S-S^{\prime}\right) d \nu$ is given as a percentage of $\int_{-\infty}^{+\infty} d \nu$ for different values of $S_{0}$ and $p$. Since the mathematical treatment of the problem leads to insoluble integrals, the values of the error have been obtained by a graphical method and are, therefore, not very accurate.

\begin{tabular}{|c|c|c|c|}
\hline$S_{0}$ & $P .364$ & 0.455 & 0.909 \\
\hline 0.155 & 0.24 & 0.41 & 1.9 \\
\hline 0.398 & 1.1 & 1.7 & 5.2 \\
\hline 0.699 & 1.6 & 2.5 & 8.6 \\
\hline
\end{tabular}

The tabulated values of the error may be very roughly taken together in the formula $\Delta=16 . S_{0} \cdot p^{2}$, though $\Delta$ is of course in reality a much more complicated function of both $S_{0}$ and $p$.

\section{A. Langseth.}

E. WALLES.

Universitetets kemiske Laboratorium,

Universitetets Institut for teoretisk Eysik, København.

Dec. 18. 